\title{
microRNA-34a/c function as tumor suppressors in Hep-2 laryngeal carcinoma cells and may reduce GALNT7 expression
}

\author{
WEI LI $^{1 *}$, HUIPING MA ${ }^{2 *}$ and JI SUN ${ }^{3}$ \\ Departments of ${ }^{1}$ Otolaryngology, Head and Neck Surgery and ${ }^{2}$ Respiration, \\ The First People's Hospital of Jining, Shandong 272000; ${ }^{3}$ Department of Otolaryngology, Head and Neck Surgery, \\ Harbin Medical University Cancer Institute and Hospital, Heilongjiang 150081, P.R. China
}

Received April 27, 2013; Accepted January 10, 2014

DOI: $10.3892 / \mathrm{mmr} .2014 .1929$

\begin{abstract}
A family of small non-coding RNAs, 22 nt in length, known as microRNAs (miRNAs), regulating $30 \%$ of all human gene expression, have been reported to be involved in the pathogenesis of a number of types of cancers, including laryngeal squamous cell carcinoma (LSCC). In the current study, miR-34a and miR-34c were observed to be downregulated in human LSCC tissues. Ectopic expression of miR-34a and miR-34c in Hep-2 cells significantly induced the cell proliferation and migration ability in vitro. UDP-N-acetyl- $\alpha-\mathrm{D}-\mathrm{gal}$ actosamine:polypeptide-N-acetylgalactosaminyltransferase 7 (GALNT7), whose expression is negatively regulated by miR-34a and miR-34c in Hep-2 cells, is confirmed to be a novel direct target gene of miR-34a and miR-34c. In conclusion, the current results suggest that miR-34a and miR-34c may function as tumor suppressors in LSCC through downregulation of GALNT7. The study of miR-34a, miR-34c and its novel target, GALNT7, may serve as novel potential makers for LSCC therapy.
\end{abstract}

\section{Introduction}

microRNAs (miRNAs) are noncoding RNA molecules that act as post-transcriptional regulators of specific messenger RNA transcripts (mRNAs), resulting in targeted degradation and suppression of gene expression. miRNAs play major roles in the normal developmental processes $(1,2)$, and their dysregulation significantly contributes to various aspects of tumorigenesis in the majority of types of cancer, negatively regulating tumor suppressor genes and oncogenes (3). Although the regulator in

Correspondence to: Wei Li, Department of Otolaryngology, Head and Neck Surgery, The First People's Hospital of Jining, No. 6 Jiankang Road, Jining, Shandong 272000, P.R. China

E-mail: liwei2013141516@163.com

*Contributed equally

Key words: miR-34a, miR-34c, laryngeal carcinoma, GALNT7, Hep-2 control of gene expression has been predicted to regulate $\sim 30 \%$ of all human genes, an increasing number of miRNAs assigned to their target mRNAs with specific functions must be excluded.

Laryngeal squamous cell carcinoma (LSCC) is one of the most common types of head and neck squamous carcinoma (HNSCC), and HNSCC is the sixth most frequent type of cancer in the world (4-6). At present, the main treatment strategy for LSCC is surgery or total laryngectomy followed by radiotherapy; however, the prognosis remains poor. Thus, to further improve the survival and remission rates, the carcinogenic mechanisms of LSCC requires further elucidation.

The majority of evidence suggested that miR-34a and miR-34c is aberrantly expressed in the majority of types of cancer, which lead to significant malignancies, including growth and invasion alteration $(7,8)$. However, the mechanism of miRNAs functioning as tumor suppressor or oncogenes in the process of tumor development is complex and varied, and may involve molecular and network regulatory pathways. Thus, the association between miR-34a and miR-34c and LSCC requires further investigation, particularly, their functional targets. In the current study, miR-34a and miR-34c were observed to be downregulated in eight human laryngeal cancer tissues compared with the relative adjacent normal tissue. Overexpression of miR-34a or miR-34c in the laryngeal cancer cell line Hep-2 may suppress cell growth with MTT and colony formation assay. Co-expression of miR-34a and miR-34c may restrict cell movement with wound healing assay. Furthermore, UDP-N-acetyl- $\alpha$-D-galactosamine:polyp eptide-N-acetylgalactosaminyltransferase 7 (GALNT7) was identified as a novel functional target of miR-34a and miR-34c in the Hep-2 laryngeal carcinoma cell line. Understanding the modulatory pathways of miR-34a and miR-34c may aid in characterizing the progression of laryngeal carcinoma.

\section{Materials and methods}

Laryngeal carcinoma samples, cell lines, transfection and RNA extraction. Human laryngeal carcinoma samples were obtained from The First People's Hospital of Jining (Jining, Shandong, China) with patients' informed consent and approval by the Ethics Committee of of the First People's Hospital of Jining. The Hep-2 laryngeal carcinoma cell line was cultured at $37^{\circ} \mathrm{C}$ with $5 \% \mathrm{CO}_{2}$ in RPMI-1640 media 
(Gibco-BRL, Grand Island, NY, USA) supplemented with 10\% fetal bovine serum (FBS). Hep-2 cells were transfected with Lipofectamine 2000 reagent (Invitrogen Life Technologies, Carlsbad, CA, USA). Enriched small RNAs were extracted using the mirVana ${ }^{\mathrm{TM}}$ miRNA Isolation kit (Ambion Inc., Austin, TX, USA). Total RNA was extracted using the TRIzol reagent (Invitrogen Life Technologies).

Cell growth assay. Cells were seeded in 96-well plates at 8,000 cells/well and transfected the following day. An MTT assay was used to determine relative cell growth 24,48 and $72 \mathrm{~h}$ following transfection. A MTT solution of $20 \mu \mathrm{l}$ was added into $100 \mu \mathrm{l}$ culture media and cells were incubated for a further $4 \mathrm{~h}$ at $37^{\circ} \mathrm{C}$. Next, the optical density was measured at $570 \mathrm{~nm}(\mathrm{~A} 570)$.

Colony formation assay. The cells were seeded into 12-well plates at a density of 200 cells/well following transfection. The medium was changed every three days. Approximately 10 days later, the majority of the cell clones contained $>50$ cells. The clones were washed with $1 \mathrm{X}$ phosphate-buffered saline (PBS) and stained with crystal violet for $\sim 5 \mathrm{~min}$. Finally, images were captured of the clones and clones were counted. The colony formation rate $=$ (no. of clones) $/$ (no. of seeded cells) $\times 100 \%$.

Wound healing assay. Cells were transfected and cultured to $>90 \%$ confluency in 24 -well dishes. A sterile $200 \mu \mathrm{l}$ pipette tip was used to scratch three separate wounds through the cells moving perpendicular to the line drawn in the step above. The medium was removed and replaced with fresh medium. Images were captured immediately above and below each line to ensure that the line appeared in each image. Two straight lines were created close to the border of the wound, and the width of the wound was defined as the vertical dimension of the two lines. The wound border was measured in nine random fields.

Quantitative polymerase chain reaction $(q P C R)$. The stem-loop qPCR method (9) was used to detect the miR-34a and miR-34c levels in Hep-2 cells and laryngeal carcinoma tissue. The detection of GALNT7 mRNA levels was in accordance with a previous study (10). The SYBR-Green Mix $\mathrm{Taq}^{\mathrm{TM}}$ kit (Takara Bio, Inc., Shiga, Japan) was used to trace the amplified DNA.

Western blot analysis. The Hep-2 cells were seeded into a 6 -well plate at a density of $3 \times 10^{5}$ cells/well and the cells were transfected when the cell density reached $\sim 80 \%$ confluency in the second day. At $48 \mathrm{~h}$ following transfection, the cells were lysed using RIPA buffer for $30 \mathrm{~min}$ at $4^{\circ} \mathrm{C}$. The protein concentration was measured by the $\mathrm{BCA}$ method and $20 \mu \mathrm{g}$ protein was loaded into SDS-PAGE for analysis. The first antibody was rabbit polyclonal anti-human GALNT7 antibody (1:1,000; Abcam, Cambridge, MA, USA) and rabbit monoclonal anti-human GAPDH antibody (1:1,000; Abcam). The second antibody was goat anti-rabbit IgG conjugated with horseradish peroxidase (1:1,000; Abcam). The bound antibodies were detected with the use of ECL Plus Western Blotting Detection system (GE Healthcare, Piscataway, NJ, USA) and the chemiluminescent signals were detected with the use of high-performance chemiluminescence film (GE Healthcare).

Luciferase reporter assay. Plasmids containing GALNT7-3'UTR and GALNT7-3'UTR mutations were constructed via technical support from Dajin Co. (Guangdong, China). 3'-UTR sequence of GALNT7 was predicted by six miRNA target prediction algorithms to interact with miR-34a, miR-34c and a mutated sequence of the 3'-UTR sequence was inserted into pGL3 vectors (Promega, Madison, WI, USA). Following transfection of miR-34a or miR-34c for $24 \mathrm{~h}$, Hep-2 cells were transfected with pGL3/GALNT7-3'UTR and pGL3/GALNT7-3'UTR mutant plasmids. After $48 \mathrm{~h}$, luciferase activity of Hep-2 cells was measured $96 \mathrm{~h}$ after transfection using the Dual-Luciferase reporter assay system (Promega).

Statistical analysis. All the data are shown as the mean \pm SD, and the difference between groups was determined by a two-tailed Student's t-test. $\mathrm{P}<0.05$ and $\mathrm{P}<0.01$ were considered to indicate a statistically significant difference.

\section{Results}

Downregulation of $m i R-34 a$ and $m i R-34 c$ in laryngeal carcinoma tissue. A previous study showed that miR-34c was downregulated in the laryngeal cancer tissue compared with normal laryngeal tissue (11) using miRNA profiling. Cai et al (8) reported that miR-34c is capable of suppressing growth and invasion of human laryngeal carcinoma cells by targeting c-Met. However, the precise expression level of miR-34a in laryngeal carcinoma tissue remains unclear. Thus, qPCR between 8 pairs of laryngeal carcinoma and adjacent non-tumor tissue was performed. As shown in Fig. 1, miR-34a and miR-34c expression was decreased compared with the adjacent non-tumor tissue. These data suggested that miR-34a and miR-34c were downregulated in laryngeal carcinoma, which implied that the two of these miRNAs play tumor suppressor roles in laryngeal carcinoma development.

miR-34a and miR-34c inhibit the growth of Hep-2 cells in vitro. MTT and colony formation assays were used to determine the effects of miR-34a and miR-34c in Hep-2 cells. The expression of miR-34a and miR-34c mimics was identified by qPCR (Fig. 2A). The mean A570 values in each group (Fig. 2B) suggest that transfection of Hep-2 cells with miR-34a or miR-34c caused suppression of Hep-2 cell proliferation at $72 \mathrm{~h}$ post-infection when compared with controls. As expected, miR-34a or miR-34c had a similar effect on the colony formation ability of Hep-2 cells as that on the cell viability. Overexpression of miR-34a reduced the colony formation ability by $\sim 64 \%$, while miR-34c led to a $58 \%$ reduction (Fig. 2C). This implied that miR-34a and miR-34c suppresses cell growth significantly in human laryngeal carcinoma.

Combination expression of $\mathrm{miR}-34 \mathrm{a}$ and $\mathrm{miR}-34 \mathrm{c}$ induces decreased cell motility of Hep-2 cells. To further investigate the effect of miR-34a and miR-34c on cell motility, a wound healing assay was measured post-infection for $0,12,24$ and 36 h in Hep-2 cells. Notably, a slight decrease (not statistically significant, data not shown) in cell motility of Hep-2 


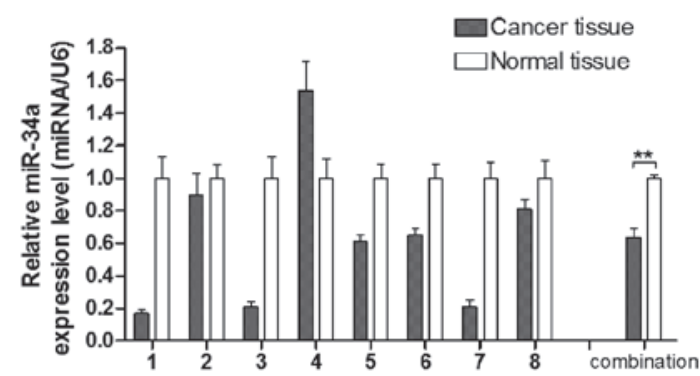

B

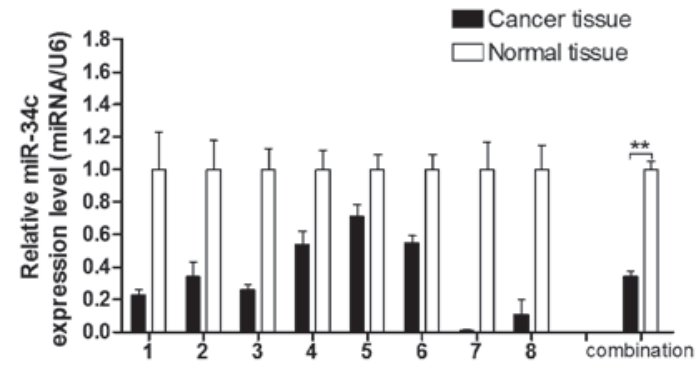

Figure 1. miR-34a and miR-34c were downregulated in laryngeal carcinoma tissues. (A) miR-34a and (B) miR-34c expression levels were analyzed by qPCR in laryngeal carcinoma tissues and adjacent non-tumor tissues. ${ }^{* * *} \mathrm{P}<0.05$, vs. control. qPCR, quantitative polymerase chain reaction.

cells either transfected with miR-34a or miR-34c alone was observed. However, as time increased, the difference of migration distance was gradually increased in co-expression of miR-34a and miR-34c groups compared with the control group (Fig. 2D). These results indicated that the combination of miR-34a and miR-34c plays an important role in Hep-2 cell motility, which may be explained by a synergism mechanism in biology. Thus, it was concluded that the combination of miR-34a and miR-34c could decrease cell motility of Hep-2 cells in human laryngeal carcinoma.

Identification of GALNT7 as a target of miR-34a and $m i R-34 c$. As miR-34a and miR-34c have pivotal functions in LSCC, the question as to how miRNA exerts its role in LSCC requires investigation. In the current study, the TargetScan algorithm was used to identify the target genes of miR-34a and miR-34c. A list of functional genes were identified, including MYCN, Cyclin D1, B-cell lymphoma 2 (BCL-2), E2F1, E2F3, CDC25A and c-Met, which have been reported to be direct targets of miR-34a or miR-34c in various types of cancers. Although a number of target genes of miR-34a and miR-34c were validated, it has been proposed that a single miRNA may target several genes and multiple miRNAs may target a single gene in a comprehensive manner $(12,13)$. Thus, the functional target of miR-34a and miR-34c in laryngeal carcinoma requires further investigation. Of the identified genes, GALNT7 was selected, which is reported to play important roles in the pathogenesis of cervical cancer and the behavior of melanoma cells $(10,14)$.

To confirm whether the 3'UTR of GALNT7 was a functional target of miR-34a and miR-34c in LSCC, multiple miRNA prediction algorithm screen methods were prepared. As shown in Fig. 3A, the table suggests that the six programs, TargetScan, miRDB, RNA22, microRNA.org, DIANA LAB and PicTar, predicted GALNT7 as a potential target of miR-34a, and each 
A

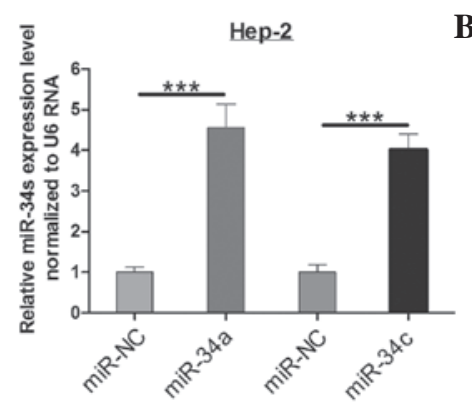

C
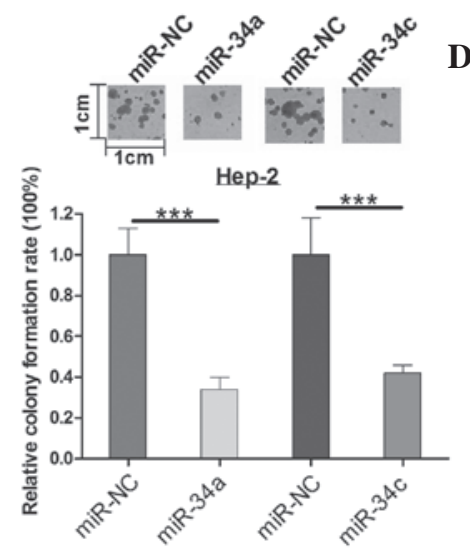

B

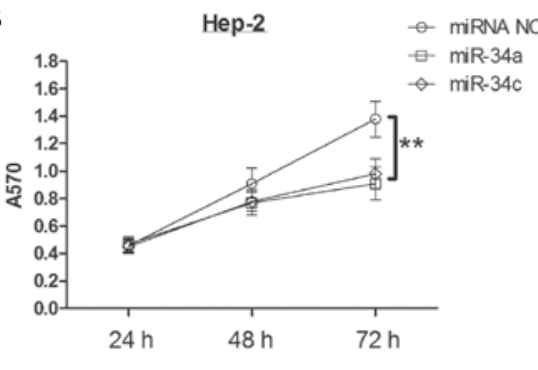

D

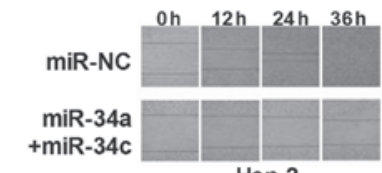

Hep-2

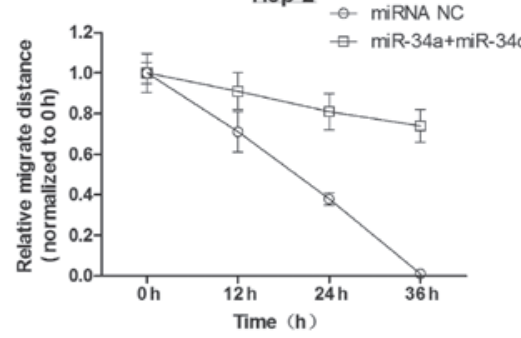

Figure 2. Effects of miR-34a and miR-34c on the cell growth and motility of Hep-2 cells. The cells were transfected with miR-34a and miR-34c together with the controls. (A) miR-34a and miR-34c expression levels were analyzed by qPCR. (B) MTT assay was performed to detect the effect of miR-34a and miR-34c on the cell viability in Hep-2 cells with 24, 48 and $72 \mathrm{~h}$ following transfection. (C) Colony formation assay was performed to detect the effect of miR-34a and miR-34c on cell growth in Hep-2 cells. The colonies were stained with crystal violet and counted. ${ }^{* * *} \mathrm{P}<0.01$, vs. control. (D) Wound healing assay was performed to detect the migration activity of Hep-2 cells co-transfected with miR-34a and miR-34c. Representative images were captured $0,12,24$ and $36 \mathrm{~h}$ following transfection. qPCR, quantitative polymerase chain reaction.

A

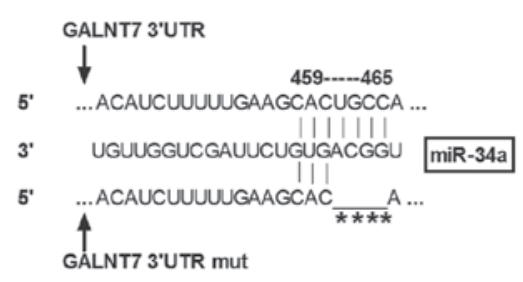

C

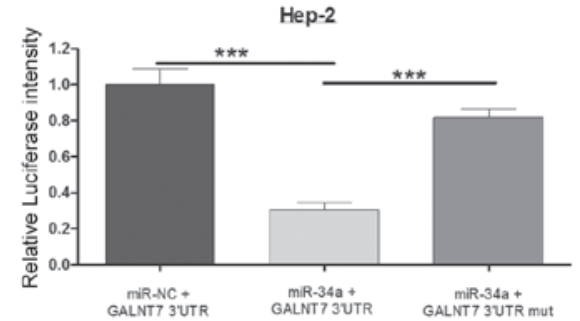

B

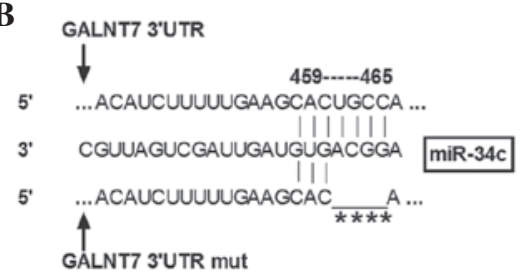

D

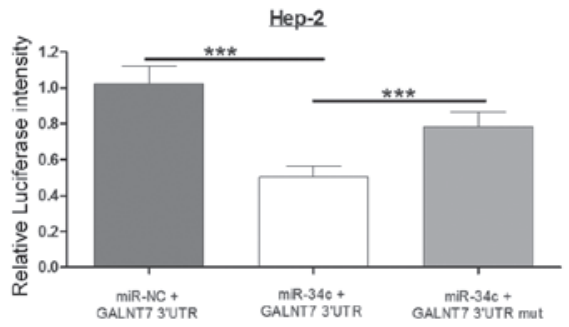

Figure 3. Prediction of GALNT7 for miR-34a and miR-34c and its validation. (A and B) Showed the algorithms between miR-34a or miR-34c and the 3'UTR of GALNT7, and also the mutant GALNT7 3'UTR (several nucleotides within binding sites were deleted). (C and D) Luciferase reporter assay was performed to detect the effect of miR-34a and miR-34c on the Luciferase intensity controlled by 3 'UTR of GALNT7. ${ }^{* * *} \mathrm{P}<0.01$, vs. control.

yielded a high score, respectively. Furthermore, four programs, TargetScan, miRDB, RNA22 and microRNA.org, predicted that miR-34c may directly target GALNT7. Next, whether miR-34a and miR-34c directly target GALNT7 in laryngeal carcinoma was investigated using a Luciferase reporter system. The alignment of miR-34a and miR-34c with the GALNT7 3'UTR insert is presented in Fig. 3B, including the seed sequence 459-465.
Thus, a mutated 3'UTR vector was constructed with four nucleotides deleted in the seed sequence (Fig. 3C). When miR-34a was overexpressed by miR-34a mimics, the Luciferase expression level was significantly lower compared with the miR-negative control group. However, the Luciferase intensity, with the mutated 3'UTR, was not affected by miR-34a (Fig. 3D). The same effect was observed when cells were transfected with 
A

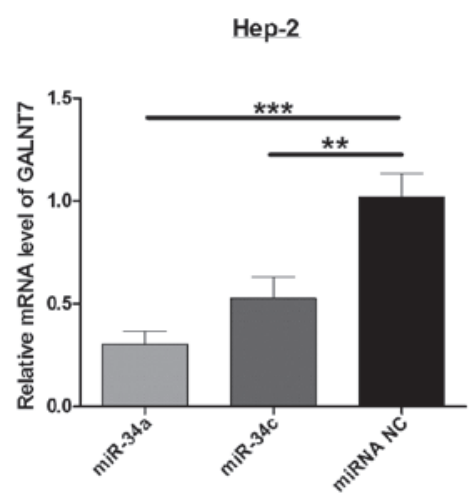

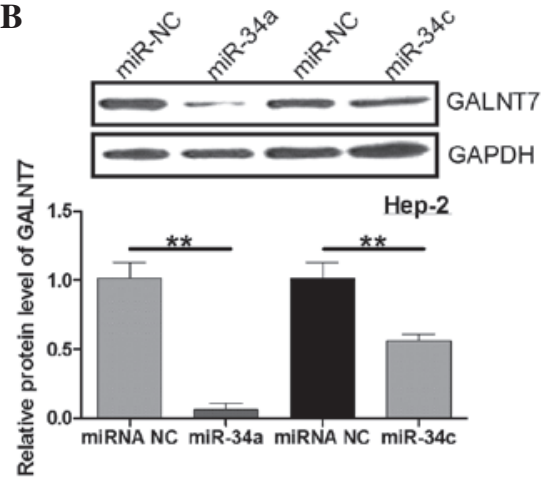

Figure 4. GALNT7 expression level in Hep-2 cells was reduced by miR-34a and miR-34c. (A) qPCR was performed to detect the effect of miR-34a and miR-34c on GALNT7 mRNA expression. (B) Western blot analysis was performed to detect the effect of miR-34a and miR-34c on the protein levels of GALNT7. ${ }^{* *} \mathrm{P}<0.05 ;{ }^{* * *} \mathrm{P}<0.01$, vs. control. qPCR, quantitative polymerase chain reaction.

miR-34c in Hep-2 cells (Fig. 3E). Thus, it was concluded that GALNT7 is a direct target of miR-34a and miR-34c in Hep-2 cells.

miR-34a and miR-34c downregulate GALNT7 expression in Hep-2 cells. Next, qPCR and western blot analysis were performed to determine the regulation of endogenous GALNT7 by miR-34a and miR-34c. Compared with the control, miR-34a and miR-34c significantly decrease GALNT7 mRNA expression, while the GALNT7 protein level was also significantly downregulated when Hep-2 cells were cotransfected with miR-34a or miR-34c ( $\mathrm{P}<0.05$; Fig. 4). These results indicate that miR-34a and miR-34c negatively regulate GALNT7 expression in the Hep-2 laryngeal carcinoma cell line.

\section{Discussion}

Over the past number of years, a large number of miRNAs have been reported to play important roles in regulating cell biology processes, including tumorigenesis, and reduce its target gene expression by mRNA degradation or mRNA cleavage $(15,16)$. The miRNAs have been confirmed as tumorigenic and tumor suppressing genes, and include miR-17-92 and let-7 $(17,18)$.

Among these functional miRNAs, miR-34a and miR-34c were firmly established to exhibit tumor suppressive effects in multiple types of cancer, including leukemias, hepatocellular carcinoma, pancreatic and colon cancer (19-22). In the present study, miR-34a and miR-34c were observed to be downregulated in human laryngeal carcinoma compared with the adjacent normal tissue. Next, transfection of Hep-2 cells with miR-34a and miR-34c was shown to significantly induce growth and migration inhibition by MTT and colony formation assays. These results confirmed the tumor suppressor role of miR-34a and miR-34c in laryngeal carcinoma. Notably, when Hep-2 cells were transfected with miR-34a and miR-34c alone, there were little changes with the movement ability of Hep-2 cells using a wound healing assay. However, co-expression of miR-34a and miR-34c significantly reduces Hep-2 cell motility. Therefore, miR-34a and miR-34c were hypothesized to affect the motility of Hep-2 cells and may cooperate with each other by targeting cell metastasis-associated genes. Thus, the functional targets of the miR-34 family in laryngeal carcinoma require further investigation.

At present, miR-34a and miR-34c have multiple experimentally validated targets involved with cellular proliferation and apoptosis, including MYCN, BCL2, SIRT1, SFRP1, CAMTA1, NOTCH1, JAG1, CCND1, CDK6 and E2F3 (21,23-29). In the current study, the GALNT7 was identified as a novel target using 6 algorithms, TargetScan, miroRNA.org, PicTar, miRDB, DIANA LAB and RNA22. These were selected because greater specificity in miRNA prediction may be attained using the consensus of multiple algorithms. Notably, the Luciferase report assay confirmed that miR-34a and miR-34c may directly target GALNT7-3'UTR with the seed sequence of 459-465 sites. The western blot analysis and qPCR assay demonstrated that the miR-34a and miR-34c also regulate endogenous GALNT7 expression in Hep-2 cells in mRNA and protein levels.

GALNT7, a member of glycosyltransferases, catalyzes the transfer of GalNAc to serine and threonine residues on target proteins, thus, initiating mucin-type O-linked glycosylation in the Golgi apparatus (30). Previous studies have reported that glycosylation may play a role in carcinogenesis and metastasis in a number of types of common cancers (31). A recent study revealed that ectopic expression of $\mathrm{miR}-30 \mathrm{~b} / 30 \mathrm{~d}$ promoted the metastatic behavior of melanoma cells, and in vitro and in vivo data implicated GALNT7 inhibition as a key contributor of the prometastatic effects of miR-30d. This miR-30d/GALNT7 axis may be involved in the regulation of the synthesis of the immunosuppressive cytokine, IL-10, and reduced immune cell activation and recruitment (14). Other miRNAs were also found to be upstream regulators of GALNT7, including miR-378 (32). In the current study, miR-34a and miR-34c were identified to regulate GALNT7 expression and directly target its 3'UTR in Hep-2 cells. To the best of our knowledge, there may be many more miRNAs that regulate GALNT7 in tumor models, thus, future studies aim to determine the number of miRNAs which directly target GALNT7, and which may support the key contribution to regulate GALNT7 repression in Hep-2 cells.

In conclusion, the expression of miR-34a and miR-34c in eight pairs of laryngeal carcinoma were evaluated, and the two were observed to be downregulated in laryngeal 
carcinoma tissue compared with their adjacent normal tissue. Overexpression of miR-34a and miR-34c alone suppressed cell growth of Hep-2 cells, and co-expressed miR-34a and miR-34c are capable of reducing the movement ability of Hep-2 cells. The phenotypic changes of Hep- 2 cells by miR-34a and miR-34c potentially occur through inhibition of GALNT7. These data suggest that the association between miR-34a and miR-34c with its target novel GALNT7 may aid in understanding the molecular mechanism of the tumorigenesis of laryngeal carcinoma.

\section{References}

1. Breving $\mathrm{K}$ and Esquela-Kerscher A: The complexities of microRNA regulation: mirandering around the rules. Int J Biochem Cell Biol 42: 1316-1329, 2010.

2. Kim VN: Small RNAs: classification, biogenesis, and function. Mol Cells 19: 1-15, 2005.

3. Garzon R, Calin GA and Croce CM: MicroRNAs in cancer. Annu Rev Med 60: 167-179, 2009.

4. Mao L, Hong WK and Papadimitrakopoulou VA: Focus on head and neck cancer. Cancer Cell 5: 311-316, 2004.

5. Licitra L, Bernier J, Grandi C, et al: Cancer of the larynx. Crit Rev Oncol Hematol 47: 65-80, 2003.

6. Vokes EE, Weichselbaum RR, Lippman SM and Hong WK: Head and neck cancer. N Engl J Med 328: 184-194, 1993.

7. Tivnan A, Tracey L, Buckley PG, Alcock LC, Davidoff AM and Stallings RL: MicroRNA-34a is a potent tumor suppressor molecule in vivo in neuroblastoma. BMC Cancer 11: 33, 2011.

8. Cai KM, Bao XL, Kong XH, et al: Hsa-miR-34c suppresses growth and invasion of human laryngeal carcinoma cells via targeting c-Met. Int J Mol Med 25: 565-571, 2010.

9. Chen C, Ridzon DA, Broomer AJ, et al: Real-time quantification of microRNAs by stem-loop RT-PCR. Nucleic Acids Res 33: e179, 2005.

10. Peng RQ, Wan HY, Li HF, Liu M, Li $X$ and Tang $H$ : MicroRNA-214 suppresses growth and invasiveness of cervical cancer cells by targeting UDP-N-acetyl- $\alpha$-D-galactosamine:pol ypeptide $\mathrm{N}$-acetylgalactosaminyltransferase 7. J Biol Chem 287: 14301-14309, 2012.

11. Liu M, Wu H, Liu T, et al: Regulation of the cell cycle gene, BTG2, by miR-21 in human laryngeal carcinoma. Cell Res 19: 828-837, 2009.

12. Peter ME: Targeting of mRNAs by multiple miRNAs: the next step. Oncogene 29: 2161-2164, 2010.

13. Hobert O: miRNAs play a tune. Cell 131: 22-24, 2007.

14. Gaziel-Sovran A, Segura MF, Di Micco R, et al: $\mathrm{miR}-30 \mathrm{~b} / 30 \mathrm{~d}$ regulation of GalNAc transferases enhances invasion and immunosuppression during metastasis. Cancer Cell 20: 104-118, 2011.
15. Lagos-Quintana M, Rauhut R, Lendeckel W and Tuschl T: Identification of novel genes coding for small expressed RNAs. Science 294: 853-858, 2001.

16. Bartel DP: MicroRNAs: genomics, biogenesis, mechanism, and function. Cell 116: 281-297, 2004.

17. Johnson SM, Grosshans H, Shingara J, et al: RAS is regulated by the let-7 microRNA family. Cell 120: 635-647, 2005.

18. Hayashita Y, Osada H, Tatematsu Y, et al: A polycistronic microRNA cluster, miR-17-92, is overexpressed in human lung cancers and enhances cell proliferation. Cancer Res 65: 9628-9632, 2005.

19. Tazawa $H$, Tsuchiya $N$, Izumiya $M$ and Nakagama $H$ : Tumor-suppressive miR-34a induces senescence-like growth arrest through modulation of the E2F pathway in human colon cancer cells. Proc Natl Acad Sci USA 104: 15472-15477, 2007.

20. Ji Q, Hao X, Zhang M, et al: MicroRNA miR-34 inhibits human pancreatic cancer tumor-initiating cells. PLoS One 4: e6816, 2009.

21. Pang RT, Leung CO, Ye TM, et al: MicroRNA-34a suppresses invasion through downregulation of Notch1 and Jagged1 in cervical carcinoma and choriocarcinoma cells. Carcinogenesis 31: 1037-1044, 2010

22. Li N, Fu H, Tie Y, et al: miR-34a inhibits migration and invasion by down-regulation of c-Met expression in human hepatocellular carcinoma cells. Cancer Lett 275: 44-53, 2009.

23. Yamakuchi M, Ferlito M and Lowenstein CJ: miR-34a repression of SIRT1 regulates apoptosis. Proc Natl Acad Sci USA 105: 13421-13426, 2008.

24. Welch C, Chen Y and Stallings RL: MicroRNA-34a functions as a potential tumor suppressor by inducing apoptosis in neuroblastoma cells. Oncogene 26: 5017-5022, 2007.

25. Wei JS, Song YK, Durinck S, et al: The MYCN oncogene is a direct target of miR-34a. Oncogene 27: 5204-5213, 2008.

26. Sun F, Fu H, Liu Q, et al: Downregulation of CCND1 and CDK6 by miR-34a induces cell cycle arrest. FEBS Lett 582: 1564-1568, 2008.

27. Bommer GT, Gerin I, Feng Y, et al: p53-mediated activation of miRNA34 candidate tumor-suppressor genes. Curr Biol 17: 1298-1307, 2007.

28. Liu H, Brannon AR, Reddy AR, et al: Identifying mRNA targets of microRNA dysregulated in cancer: with application to clear cell renal cell carcinoma. BMC Syst Biol 4: 51, 2010.

29. Luan S, Sun L and Huang F: MicroRNA-34a: a novel tumor suppressor in p53-mutant glioma cell line U251. Arch Med Res 41: 67-74, 2010.

30. Ten Hagen KG, Fritz TA and Tabak LA: All in the family: the UDP-GalNAc:polypeptide $\mathrm{N}$-acetylgalactosaminyltransferases. Glycobiology 13: 1R-16R, 2003.

31. Casey RC, Oegema TR Jr, Skubitz KM, Pambuccian SE, Grindle SM and Skubitz AP: Cell membrane glycosylation mediates the adhesion, migration, and invasion of ovarian carcinoma cells. Clin Exp Metastasis 20: 143-152, 2003.

32. Kahai S, Lee SC, Lee DY, et al: MicroRNA miR-378 regulates nephronectin expression modulating osteoblast differentiation by targeting GalNT-7. PLoS One 4: e7535, 2009. 